\title{
INTEGRATING REMOTE SENSING AND ANCILLARY DATA FOR REGIONAL ECOSYSTEM ASSESSMENT: EUCALYPTUS GRANDIS AGRO-SYSTEM IN KWAZULU-NATAL, SOUTH AFRICA
}

\author{
Moses Cho ${ }^{1}$, Jan van Aardt ${ }^{2}$, Russell Main ${ }^{1}$, Bongani Majeke ${ }^{1}$, Abel Ramoelo ${ }^{1}$, Renaud Mathieu ${ }^{1}$, Mark Norris-Rogers ${ }^{3}$, Marius Du \\ Plessis $^{3}$ \\ ${ }^{1}$ Council for Scientific and Industrial Research (CSIR), Natural Resources and the Environment, Ecosystems- Earth Observation, P.O. Box \\ 395, Pretoria, 0001, South Africa. \\ ${ }^{2}$ RIT: Center for Imaging Science, Laboratory for Imaging Algorithms and Systems \\ 54 Lomb Memorial Drive, Building 17-3173, Rochester NY 14623, USA \\ ${ }^{3}$ Mondi S.A., P O Box 39, Pietermaritzburg, 3200
}

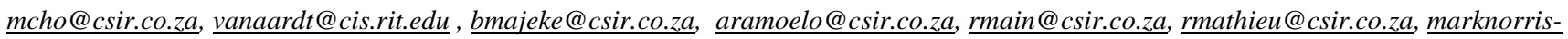
rogers@mondigroup.com, Marius.duPlessis@mondigroup.com,

\begin{abstract}
The ability of various ecosystems to perform vital functions such as biodiversity production, and water, energy and nutrient cycling depends on the ecosystem state, i.e. health. Ecosystem state assessment has been a topic of intense research, but has reached a point at which accurate large scale (e.g. regional to global scale) modelling and monitoring are hindered by limitations in conventional assessment methods such as direct field sampling, modelling from environmental drivers such as temperature, precipitation and available nutrients, and modelling from remote sensing data. The Ecosystem-Earth Observation (Eco-EO) research group at the Council for Scientific and Industrial Research (CSIR), South Africa has highlighted the need in remote sensing research for an integrated sensing approach at the systems level. This perspective is based on the assumption that a modelling approach that exploits the strength of the various techniques (in situ environmental variables, direct field observation and remote sensing data) could potentially improve the assessment of ecosystem state at various geographic scales. In this light, the EcoEO research group has embarked on an agro-system state assessment project since 2007 as a first step towards the implementation of the integrated modelling approach for various ecosystems. The agro-system consists of a monoculture forest plantation of Eucalyptus grandis situated in KwaZulu-Natal, South Africa. This paper presents preliminary results from the KwaZuluNatal E. grandis experimental study.
\end{abstract}

Keywords: Hyperspectral remote sensing, spectral indices, forestry

\section{INTRODUCTION}

Ecosystem functions including biodiversity production, and water, energy and nutrient cycling are essential to human civilisation[1]. The ability of various ecosystems to perform the above functions depends on the ecosystem state, i.e. health[2]. Therefore, effective monitoring of ecosystem state including the structure, species composition, canopy biochemistry and productivity is crucial to ensuring the well-being of various ecosystems. Ecosystem state assessment has been a topic of intense research, but has reached a point at which accurate large scale (e.g. regional to global scale) modelling and monitoring are hindered by limitations in conventional assessment techniques such as direct field sampling, modelling from environmental drivers such as temperature, precipitation and available nutrients, and modelling from remote sensing data (Figure 1).

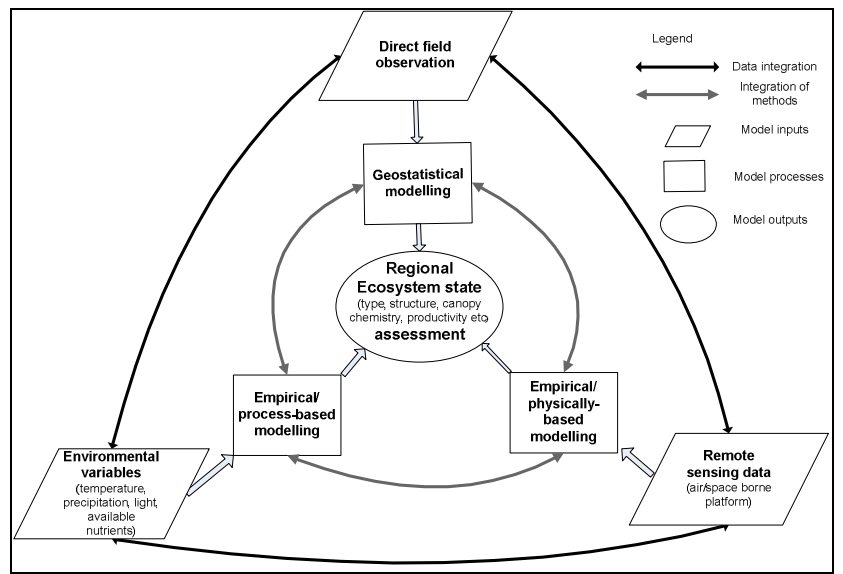

Figure 1. Conceptual framework

First, direct field techniques for quantifying ecosystem state are difficult, extremely labour intensive, and costly in terms of time and money. Furthermore, they cannot usually be extended over regional scales of vegetation because of logistics and huge number of sample variations[3]. Second, it has long been established that environmental variables including climatic, edaphic and topographic factors determine ecosystem state at various scales[4, 5]. These environmental variables have either been used empirically to estimate and map ecosystem state or as input variables in ecosystem process models[6]. Ecosystem process models applied in a spatially distributed mode can assimilate and integrate a diverse assemblage of environmental data[6]. However, the draw back of this approach is that some ecological phenomena are stochastic and cannot be predicted by environmental variables alone e.g. fire, grazing, other anthropogenic factors. Lastly, remote sensing, using current or anticipated technology, is widely viewed as a non-subjective method for large-scale monitoring of ecosystem state and processes. Remote sensing has been used extensively to quantify vegetation biophysical and biochemical properties[3]. However, reported estimation accuracies are low for 
ecosystems characterised by subtle differences in biophysical and biochemical variables[3].

The Ecosystem-Earth Observation (Eco-EO) research group at the Council for Scientific and Industrial Research (CSIR) has highlighted the need in remote sensing research for an integrated sensing approach at the systems level. This perspective is based on the assumption that a modelling approach that exploits the strength of the various techniques (in situ environmental variables, direct field observation and remote sensing data) could potentially improve the assessment of ecosystem state at various geographic scales. Figure 1 illustrates the general concept of system-level integrated modelling. Integration can occur at two level; data and modelling techniques. For example, (i) empirical models can be derived from integrating remote sensing and environmental data (e.g.[7]) (ii) using direct field observations as the main data and remote sensing or environmental variables as co-variables, ecosystem state can be estimated by geostatistical modelling (e.g.[8]), (iii) remote sensing metrics can be used as input parameters in process models of plant growth e.g. [6], (iii) environmental and field data can be used to define radiative transfer models for canopy reflectance ( e.g. [9]).

The Eco-EO research group has embarked on an agro-system state assessment project since 2007 as a first step towards the implementation of the integrated modelling approach for various ecosystems. The agro-system consists of a monoculture forest plantation of Eucalyptus grandis situated in KwaZulu-Natal, South Africa. This experimental site is classified according to three site qualities namely good, medium, and poor based on soil quality, as defined by total available water (TAW) in the soil profile. The latter is largely dependent on soil properties such as effective rooting depth and soil type, in conjunction with rainfall and temperature classes[10]. The objectives were to:

- $\quad$ assess the relationship between $E$. grandis site quality and canopy reflectance,

- develop integrated models involving remote sensing, site quality and topographic variables for estimating ecosystem state,

- parameterise radiative transfer models (leaf and canopy reflectance models) for estimating $E$. grandis ecosystem state variables

- and lastly, develop integrated models involving forest growth model and remote sensing data to predict forest productivity.

Here we present a synopsis of some of the results for the first three objectives.

\section{MATERIALS AND METHODS}

\subsection{Study site}

The study area is located in the Richmond area of the KwaZuluNatal Midlands of South Africa $\left(29^{\circ} 49^{\prime} \mathrm{S}, 30^{\circ} 17^{\prime} \mathrm{E}\right)$ and consists of 8 year old E. grandis plantations (Figure 2). Plantation forestry is a major land use in the study area due to suitable climate and soils. The terrain generally consists of undulating plains, which are incised with steep river valleys, with altitude rising from $800 \mathrm{~m}$ to $1400 \mathrm{~m}$ above-mean-sea-level. The geology consists of sandstone and clay formations, which have resulted in sandy clay, to sandy clay loam soils. The experimental site is classified according to three site qualities namely good, medium, and poor based on soil quality, as defined by total available water (TAW) in the soil profile. The latter is largely dependent on soil properties such as effective rooting depth and soil type (form), in conjunction with rainfall and temperature classes[10].

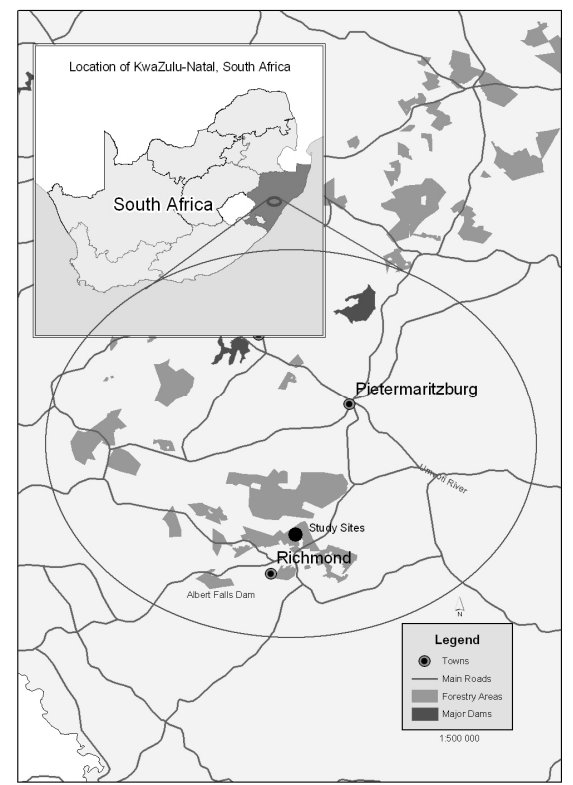

Figure 2. Study site in the Richmond area of the KwaZulu-Natal Midlands, South Africa

\subsection{Spectral and other measurements}

Two sets of measurements were made in August 2007 and August 2008. In August 2007, canopy reflectance spectra for sixty-eight trees $(25=$ good, $25=$ medium and $18=$ poor sites $)$ were collected on clear sky days using an ASD spectroradiometer (350-2500 nm) from a raised platform, while leaf and canopy spectral measurements were made for $65 \mathrm{E}$. grandis trees (15=good, $26=$ medium and $21=$ poor sites) at 19 locations on clear sky days in August 2008. For all spectral measurements, the radiance data were converted to reflectance using a reference reading (denominator) for each sample from a white spectralon reference panel. About 25 to 30 sunlit leaf samples were collected for leaf spectral and chlorophyll measurements.

For the purpose of parameterisation of leaf radiative transfer models, leaf spectra and biophysical/biochemical properties were measured for leaves at different stages of development in order to ensure a high variability in the data set. Leaf chlorophyll content data was measured using the chlorophyll-meter, SPAD-502. The SPAD readings were later converted to chlorophyll content using equations developed by Uddling et al. [11]

\subsection{Data analysis}

This section is outlined according to the objectives

- One-way analysis of variance (ANOVA) was used to assess the relationship between site quality and canopy spectral properties. The spectral proxies (spectral indices) of leaf water (normalised water difference index 

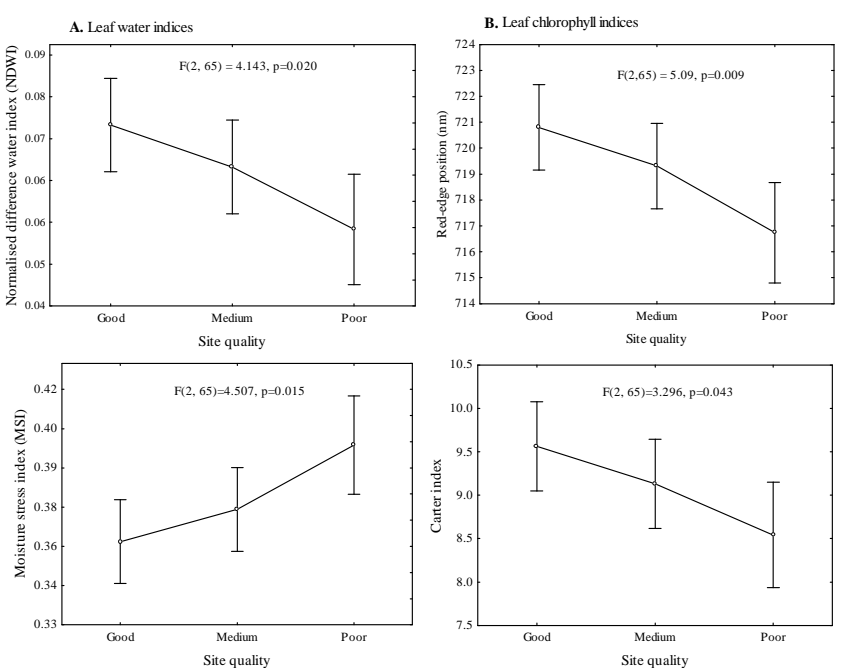

Figure 3. Relationship between site quality and remote sensing indices

- (NDWI), moisture stress index (MSI)) and chlorophyll (Carter chlorophyll index, red-edge position (REP)), content were used to describe the canopy spectral characteristics.

- With regards to assessing the utility of the integrated approach, the research method consisted of first investigating the performance of linear regression models derived from remote sensing or in situ data only and subsequently assessing if the integration of the two data types improves the estimation of an ecosystem state variable such as leaf chlorophyll content.

- As a first step towards developing a canopy reflectance model for predicting E. grandis state, we assessed the utility of the leaf radiative transfer model (RTM) in predicting E. grandis state. Leaf biophysical and biochemical parameter ranges for $E$. grandis were used to parameterise the PROSPECT RTM. PROSPECT was subsequently used to simulate 1200 leaf spectra. Remote sensing models were developed from the simulated data and inverted to estimate leaf chlorophyll content.

\section{RESULTS}

\subsection{Predicting site quality}

ANOVA results for August 2007 showed that spectral differences between site qualities are significant. Leaf water (NDWI, MSI) and chlorophyll (Carter chlorophyll index, REP) indices exhibited significant differences between sites (Figure 3). These results were not consistent with those obtained in August 2008 as spectral differences between sites in 2008 were not significant. However, REP (a leaf chlorophyll indicator) showed a non-significant gradient from the low to the good sites. Rainfall data of the study area showed 2008 (858.8 mm between September 2007-August 2008) as a wetter year than 2007 (807.2 mm between September 2006-July 2007). Although TAW was not specifically measured for the above periods, we hypothesise that the TAW gradient is most apparent in a drier year than in a wetter year.

\subsection{Integrating remote sensing and in situ data to predict Eucalyptus leaf state}

The next question was whether the integration of site quality and remote sensing data could improve the estimation of an ecosystem state variable such as leaf chlorophyll content? Leaf chlorophyll data was only available for 2008. The best leaf chlorophyll index, the red-edge position accounted for $39 \%$ of the variance in SPAD readings (Figure 4). REP and site quality were the most significant variables selected in a stepwise regression involving remote sensing indices and ancillary data (site quality and digital terrain model derived variables) for estimating leaf chlorophyll content. This integrated approach yielded an $\mathrm{R}^{2}$ of $0.47(\mathrm{p}<0.05)$. These results are significant given the subtle differences or low variability in the chlorophyll content (SPAD range $=41.4-56.3$, standard deviation $=3.3$ ) for the study site.
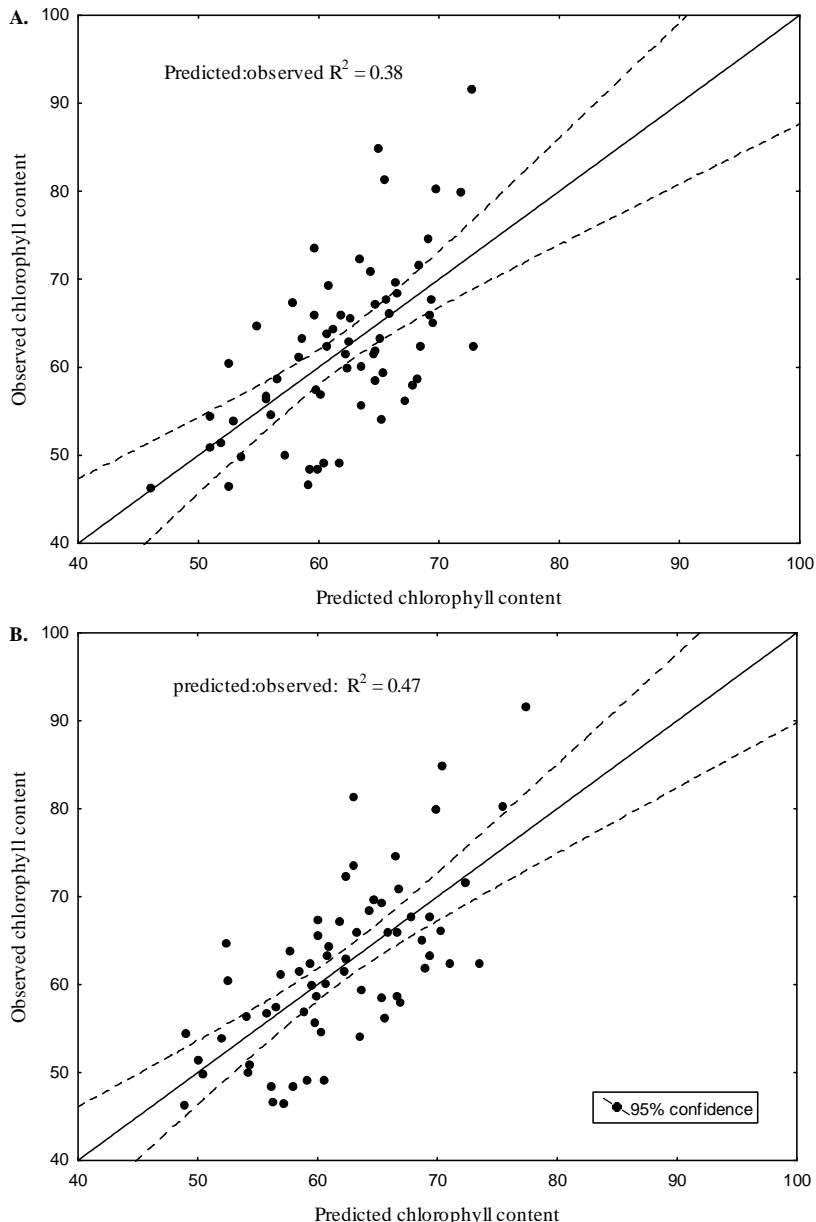

Figure 4. Relationship between predicted and observed chlorophyll

\subsection{Estimating leaf Chlorophyll using the leaf radiative transfer model - PROSPECT}

Models developed from simulated E. grandis spectra using the PROSPECT radiative transfer model yielded high accuracies for 
predicting leaf chlorophyll content. The best predictive model involved REP derived by the linear extrapolation method of Cho and Skidmore[12], root mean square error $=2 \mu \mathrm{g} \mathrm{cm} \mathrm{cm}^{-2}$, representing $4 \%$ of the mean, see Figure 5 ).

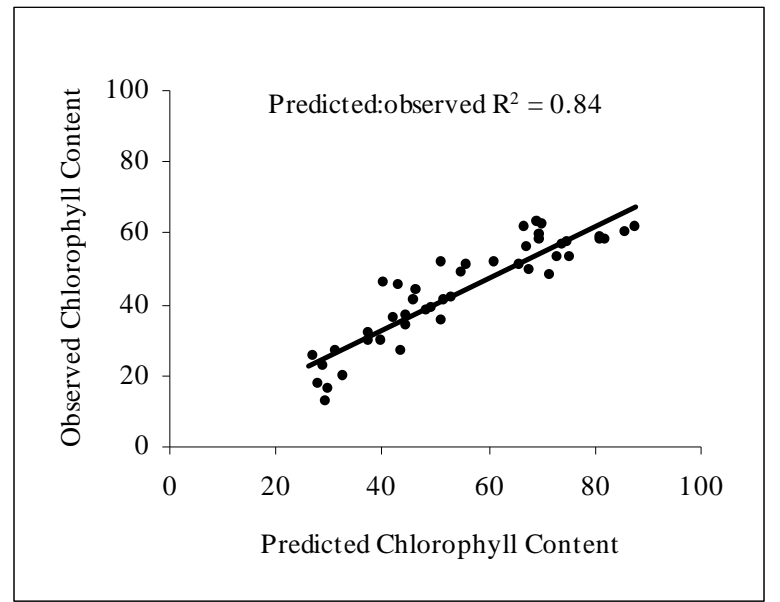

Figure 5. Relationship between predicted and observed chlorophyll from radiative transfer modelling

\section{CONCLUSIONS}

The study thus demonstrated that the integration of remote sensing and in situ data could be important in providing more accurate estimates of $E$. grandis state in KwaZulu Natal, South Africa compared to remote sensing only models. Such modelling effort would become important at the regional scale in the near future as space-borne imaging spectroscopy or hyperspectral sensors become more available. The potential for developing a radiative transfer function for predicting E. grandis at the leaf level has also been demonstrated. This effort needs to be up-scaled to the canopy level.

\section{Acknowledgments}

The authors would like to thank Mondi Business Paper and the Council for Scientific and Industrial Research for respectively providing logistical and funding support for this project. We would also like to thank Dr. Mark Norris-Rogers and Mr. Marius du Plessis (Mondi BP) for their assistance during the field data collection.

\section{References}

[1] P. J. Curran, "Imaging spectrometry for ecological applications," JAG, vol. 3, pp. 305-312, 2001.

[2] G. A. Blackburn, "Quantifying Chlorophylls and Caroteniods at Leaf and Canopy Scales: An Evaluation of Some Hyperspectral Approaches," Remote Sensing of Environment, vol. 66, pp. 273-285, 1998.

[3] M. A. Cho, "Hyperspectral remote sensing of biochemical and biophysical parameters: The derivative red-edge "double-peak feature", a nuisance or an opportunity?," in Resource Ecology, vol. PhD. Wageningen: Wageningen University, 2007, pp. 206.

[4] A. M. Lafta and J. H. Lorenzen, "Effect of High Temperature on Plant Growth and Carbohydrate
Metabolism in Potato," Plant Physiol. , vol. 109, pp. 637643, 1995.

[5] T. O. Veteli, R. K. Kuokkanen, H. Julkunen-Tiitto, and R. J. Tahvanainen, "Effects of elevated CO2 and temperature on plant growth and herbivore defensive chemistry," Global Change Biology, vol. 8, pp. 12401252, 2002.

[6] D. P. Turner, S. V. Ollinger, and J. S. Kimball, "Integrating remote sensing and ecosystem process models for landscape- to regional-scale analysis of the carbon cycle," Bioscience, vol. 54, pp. 573-582, 2004.

[7] N. C. Coops, M. A. Wulder, and J. C. White, "Integrating remotely sensed and ancillary data sources to characterize a mountain pine beetle infestation," Remote Sensing of Environment, vol. 105, pp. 83-97, 2006.

[8] O. Mutanga and D. Rugege, "Integrating remote sensing and spatial statistics to model herbaceous biomass distribution in a tropical savanna," International Journal of Remote Sensing, vol. 27, pp. 3499 - 3514, 2006.

[9] R. Darvishzadeh, A. Skidmore, M. Schlerf, and C. Atzberger, "Inversion of a radiative transfer model for estimating vegetation LAI and chlorophyll in a heterogeneous grassland," Remote Sensing of Environment, vol. 112, pp. 2592-2604, 2008.

[10] C. W. Smith, R. N. Pallett, R. A. W. Gardner, and M. Du Plessis, "A Strategic forestry site classification for the summer rainfall region of southern Africa based on climate, geology and soils," in Institute for Commercial Forestry Research (ICFR), Pietermaritzburg, South Africa, Bulletin Series, vol. 03, 2005.

[11] J. Uddling, J. Gelang-Alfredsson, K. Piikki, and H. Pleijel, "Evaluating the relationship between leaf chlorophyll concentration and SPAD-502 chlorophyll meter readings," Photosynth Res vol. 91, pp. 37-46, 2007.

[12] M. A. Cho and A. K. Skidmore, "A new technique for extracting the red edge position from hyperspectral data: The linear extrapolation method," Remote Sensing of Environment, vol. 101, pp. 181-193, 2006. 